\title{
Adaptive Finite-Time Disturbance Observer Based Sliding Mode Control for Dual-Motor Driving System
}

\author{
Tianyi Zeng $\mathbb{D},{ }^{1}$ Xuemei Ren $\mathbb{D},{ }^{1}$ and Yao Zhang $\mathbb{D}^{2}$ \\ ${ }^{1}$ School of Automation, Beijing Institute of Technology, Beijing 100081, China \\ ${ }^{2}$ Queen Mary University of London, London E1 4NS, UK \\ Correspondence should be addressed to Xuemei Ren; xmren@bit.edu.cn
}

Received 26 June 2018; Accepted 14 August 2018; Published 27 September 2018

Academic Editor: Zhile Yang

Copyright ( $) 2018$ Tianyi Zeng et al. This is an open access article distributed under the Creative Commons Attribution License, which permits unrestricted use, distribution, and reproduction in any medium, provided the original work is properly cited.

\begin{abstract}
This paper investigates a precise tracking control method based on an adaptive disturbance observer for the dual-motor driving system. The unknown matched disturbance is fully considered and estimated in this paper, and the estimation error is proven to be finite-time convergent. A sliding mode controller based on the multiple sliding surface technique is proposed in which the disturbance is compensated. The overall system containing both the observer and the controller is proven to be stable. The tracking error is within the neighbourhood of the origin before the observer completes its convergence and converges to zero thereafter. Simulation results verify the effectiveness of the disturbance observer and the sliding mode controller.
\end{abstract}

\section{Introduction}

In the past decades, the motor driving system has found its application from industry to military. Due to the existence of nonlinearities caused by the friction, transmission gears, and model uncertainties, the satisfactory control performance cannot be guaranteed by linear control methods [1]. To improve the control performance of the motor driving system, many advanced control method has been adopted, such as adaptive control [2] and robust control [3]. In addition, although the advanced controller is with the robustness, disturbances caused by model uncertainties and external environments still have significant influence on the control precision. For the load tracking control problem on the motor driving system, the main concerns are the stability, the tracking accuracy, and the robustness subject to disturbances. To ensure a precisely tracking performance, model uncertainties, measurement errors, and external disturbances should be fully considered.

Due to the demand of the driving force, a multimotor driving strategy is adopted in the tracking control problem of the large inertia system, such as radar control systems, crane control systems, et al. However, the load tracking control researches are mostly focused on the single-motor driving scheme [4-6]. The control performance can be degraded if the single motor-based control method is adopted directly in the multimotor driving system due to the potential motor collision caused by existence of the synchronization problem between driving motors. To achieve the synchronization of multiaxis motions, a cross-coupled strategy was proposed in [7]. Sun developed an adaptive control scheme including cross-coupled strategy-based synchronization to guarantee the convergence of both synchronization and position errors of multiaxis motions [8]. An observer-based synchronization controller is presented for two coupling permanent magnet synchronous motors system in [9] with the adoption of the cross-coupled strategy. However, most research results focus on the motor control without considering the load tracking problem. To achieve the precise control performance of the dual-motor driving system, a cross-coupled synchronization strategy is developed with the consideration of load tracking controller design.

Finite-time or fixed-time convergence theory have been widely used in the controller and the observer design to achieve the satisfactory performance [10]. Several researches based on sliding mode control have been done to tackle the disturbance and the uncertainty [11-14]. In the first category, a switching function is designed to keep the system state 


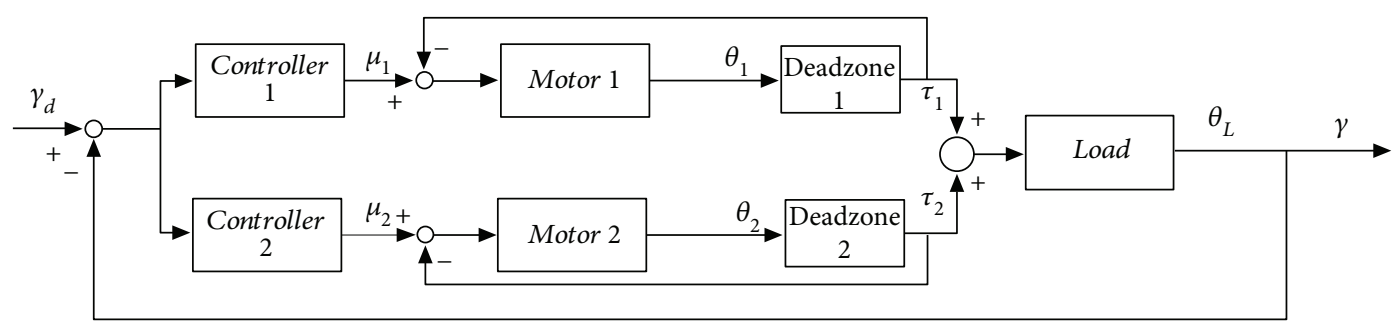

FIGURE 1: Block diagram of the dual-motor driving system.

switching repeatedly around the equilibrium point. This kind of high-frequency chattering can offset and compensate the disturbance. Furfaro and Wibben proposed a multiple surface sliding mode guidance for the landing on asteroids missions, which is proven to be finite-time stable and effective to cope with unknown environmental uncertainties [15]. Song et al. designed a finite-time controller for the nonlinear spacecraft attitude system, which applied fast terminal sliding mode control to ensure a fast transient response [16]. Guo and Zhou proposed an active disturbance rejection control method for multidimensional boundary control wave equation, in which the disturbance is compensated in the feedback loop together with a collocated stabilizing controller [17]. These methods are basically proven effective by assuming the upper limit of the disturbance is known. However, this assumption is not realistic and the actuator can hardly achieve high-frequency outputs in practical applications. Therefore, to achieve a better performance on tackling unknown disturbances, not only the upper bound of the disturbance but also its detailed information should be estimated.

In the second category, the disturbance at each time instant is estimated online by designing disturbance observers. Chen et al. proposed a nonlinear disturbance observer for the robotic manipulator system, which is exponentially stable [18]. Sariyildiz and Ohnishi designed a reaction torque disturbance observer which enhances the robustness and the overall system is proven to be stable [19]. Chen et al. proposed a disturbance observer for multi-input and multioutput (MIMO) cascade systems and designed a robust-constrained controller to address the input saturation problem [20]. Xu et al. investigated a fuzzy controller for the unknown dead zone system based on the disturbance observer, and the controller updates its fuzzy law according to the disturbance estimation [21]. Mobayen and Javadi designed a terminal sliding mode controller for a third-order chainedform nonholonomic system based on a finite-time stable disturbance observer, and the closed-loop system is proven to be finite-time convergent [22]. However, by using these methods, the disturbance is usually assumed to be slowly varying so that the time derivative of the disturbance is sufficiently small to be ignored. Since this assumption is not applicable for the motor driving system, it is necessary to investigate an adaptive observer to estimate the known disturbance online without assuming that the disturbance is bounded by a known constant or assuming that the disturbance is slowly varying.

Main contributions of this paper are as follows:
(1) An adaptive disturbance observer is designed based on the sliding mode method, which is proven to be finite-time stable

(2) The tracking and synchronization controller is designed by applying the multiple sliding surfaces technique

(3) The overall system is proven to be stable, and the tracking error can be driven within the neighbourhood of the origin before the estimation error converges to zero. After the disturbance observer completes its convergence, the load can track the desired trajectory with a high accuracy

The rest of this paper is organized as follows. The dualmotor driving system is introduced and its state space model is given in Section 2. The adaptive disturbance observer is designed in Section 3, in which the finite-time stability of the observer is given. The controller based on the multiple surface technique is proposed in Section 4. With the proposed observer and controller, the stabilization for the overall system is proven. Section 5 gives the simulation results on the disturbance observer and the controller, which verify the effectiveness of this paper. Finally, we conclude this paper in Section 6.

\section{Problem Formulation}

In this section, we consider the position tracking control problem of the dual-motor driving system. Dynamic models for both the single-motor driving system and the dual-motor driving system are established and simplified for the controller and the state observer design.

2.1. Dual-Motor Driving Scheme. The block diagram of the dual-motor driving system is shown in Figure 1. The dynamic model of the dual-motor driving system is

$$
\left\{\begin{array}{l}
J_{\mathrm{m}} \ddot{\theta}_{i}+b_{\mathrm{m}} \dot{\theta}_{i}=u_{\mathrm{i}}-\tau_{i}, \\
J_{\mathrm{L}} \ddot{\theta}_{\mathrm{L}}+f_{L}\left(\dot{\theta}_{\mathrm{L}}\right)=\sum_{i=1}^{2} \tau_{i} .
\end{array}\right.
$$

Here, $i=1,2$ represent two driving motors; $\theta_{i}$ are angular positions of the two driving motors; $\theta_{\mathrm{L}}$ is the angular position of the load; $J_{\mathrm{m}}$ and $J_{\mathrm{L}}$ are moments of inertia of driving motors and the load; $u_{\mathrm{i}}$ are control inputs of the two motors; $b_{\mathrm{m}}$ is the viscous friction coefficient of motor; $f_{L}\left(\dot{\theta}_{L}\right)$ is the friction 


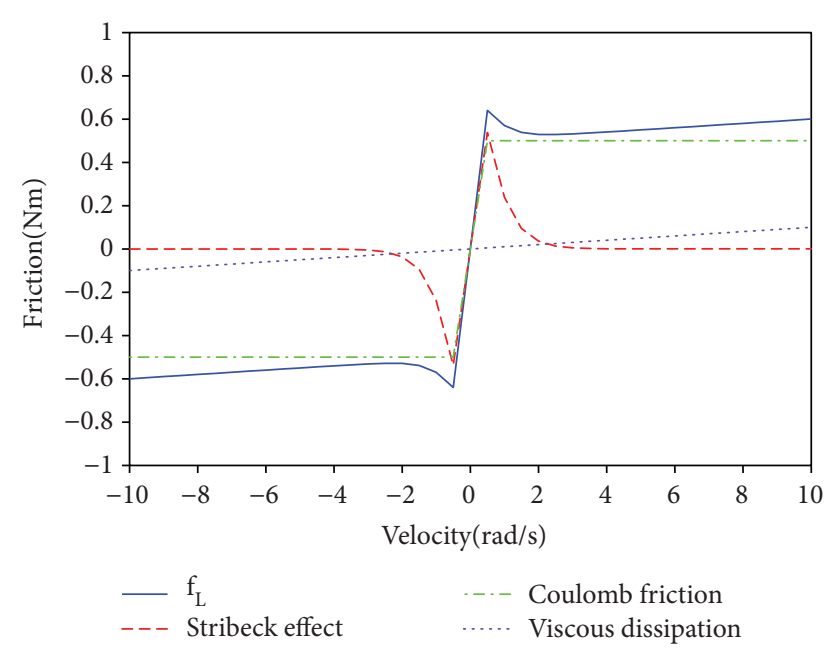

Figure 2: Friction model.

torque; $\tau_{i}$ represent transmission torques between the two motors and load and expressed as

$$
\tau_{i}=k f\left(\delta_{i}\right)+c f\left(\dot{\delta}_{i}\right)
$$

where $\delta_{i}=\theta_{i}-\theta_{L} ; k$ and $c$ are the torsional coefficient and the damping coefficient. A continuously differentiable friction model is adopted in this paper, where the friction torque can be expressed as

$$
\begin{aligned}
f_{L}\left(\dot{\theta}_{L}\right)= & A_{1}\left(\tanh \left(\rho_{1} \dot{\theta}_{L}\right)-\tanh \left(\rho_{2} \dot{\theta}_{L}\right)\right) \\
& +A_{2} \tanh \left(\rho_{3} \dot{\theta}_{L}\right)+A_{3} \dot{\theta}_{L}
\end{aligned}
$$

where $A_{1}, A_{2}, A_{3}, \rho_{1}, \rho_{2}$, and $\rho_{3}$ are positive parameters. $A_{1}$ and $A_{2}$ stand for static friction coefficients; the Stribeck effect is described by $\tanh \left(\rho_{1} \dot{\theta}_{L}\right)-\tanh \left(\rho_{2} \dot{\theta}_{L}\right) ; A_{2} \tanh \left(\rho_{3} \dot{\theta}_{L}\right)$ stands for the coulomb friction; and $A_{3} \dot{\theta}_{L}$ is the viscous dissipation. Define the parameters as $A_{1}=0.25, A_{2}=0.5, A_{3}=$ $0.01, \rho_{1}=100, \rho_{2}=1$, and $\rho_{3}=100$ [23]. The friction model is shown in Figure 2.

2.2. Single-Motor Driving System. The dual-motor driving scheme is developed based on the single-motor driving system. To simplify the controller design, a control-oriented model for single-motor driving system is established in this section.

$$
\left\{\begin{array}{l}
J_{m} \ddot{\theta}_{m}+b_{m} \dot{\theta}_{m}=u-\tau, \\
J_{L} \ddot{\theta}_{L}+f_{L}\left(\dot{\theta}_{L}\right)=\tau .
\end{array}\right.
$$

Here, $\theta_{m}$ is the angular position of the driving motor; $u$ is the control input; $\tau$ is the transmission torque of the gear box can be expressed as

$$
\tau=k f(\delta)+c f(\dot{\delta})
$$

Here, $\delta$ is the angle position error between the motor and the load, i.e., $\delta=\theta_{m}-\theta_{L} ; f(\delta)$ is a function for a deadzone:

$$
f(\delta)= \begin{cases}\delta-d_{c}, & \delta \geq d_{c} \\ 0, & |\delta|<d_{c} . \\ \delta+d_{c}, & \delta \leq-d_{c}\end{cases}
$$

Here, $d_{c}$ is the gear clearance width parameter. By ignoring the deadzone nonlinearity, there is a proportional relationship that $\theta_{m}=n \theta_{L}$ with $n$ is the gear ratio [24]. To simplify the controller design, the system (4) can be replaced by the following form.

$$
\left(n J_{m}+J_{L}\right) \ddot{\theta}_{L}+n b_{m} \dot{\theta}_{L}+f_{L}\left(\dot{\theta}_{L}\right)=u
$$

Choose system states as $x_{1}=\theta_{\mathrm{L}}$ and $x_{2}=\dot{\theta}_{L}$; the statespace equation can be expressed as follows:

$$
\left\{\begin{array}{l}
\dot{x}_{1}=x_{2}, \\
\dot{x}_{2}=J\left(u-n b_{m} x_{2}-f_{L}\left(x_{2}\right)\right)+d,
\end{array}\right.
$$

where $J=1 /\left(n J_{m}+J_{L}\right)$ and $d$ denotes the uncertainty caused by the model reduction and other external disturbances. With (8), the tracking controller can be developed for each driving motor. The synchronization problem will also be taken into consideration in the control scheme. In this paper, we aim to develop a disturbance observer based control scheme to achieve the precision load tracking control.

\section{Adaptive Disturbance Observer}

In this section, the adaptive sliding mode observer (ASMO) is proposed, in which an adaptive law and a sliding variable are designed to track the variation of the actual excitation force. The main idea of the adaptive disturbance observer (ADO) is to design a sliding variable that connects the estimated disturbance with the system state. The adaptive law is designed in the form of the super-twisting structure. Unlike other sliding mode observers, the proposed observer does not use the information of the upper bound of the disturbance but only assume that the disturbance is bounded with unknown boundary.

The proposed ADO is as follows:

$$
\left\{\begin{array}{l}
\sigma=x_{2}+h, \\
\dot{h}=-J\left(u-\mathrm{n} b_{m} x_{2}-f_{L}\left(x_{2}\right)\right)-\widehat{d}, \\
\widehat{d}=L_{1}|\sigma|^{1 / 2} \operatorname{sign}(\sigma)+L_{2} y, \\
\dot{y}=\operatorname{sign}(\sigma),
\end{array}\right.
$$


where $\sigma$ is the sliding variable and $h$ and $y$ are internal variables with no physical meanings. The gain parameters $L_{1}$ and $L_{2}$ are designed as

$$
\left\{\begin{array}{l}
L_{1}=\left|x_{2}\right||\sigma|^{2} \\
L_{2}=\frac{\varepsilon}{2} L_{1}+\frac{1}{2} \varepsilon^{2}+\frac{1}{2} \lambda
\end{array}\right.
$$

where $\varepsilon, \lambda>0$ are constants.

Theorem 1. Suppose that the disturbance is bounded, and there always exist a positive constant $\delta$ such that $|d|<\delta$ $|\sigma|^{1 / 2}$ holds. For the system (8), the estimated disturbance $\widehat{d}$ converges to the actual disturbance $d$ in a finite time by the proposed ADO (9).

Proof 1. From (8) and (9), the time derivative of the sliding variable $\sigma$ can be written as

$$
\dot{\sigma}=\dot{x}_{2}+\dot{h}=d-L_{1}|\sigma|^{1 / 2} \operatorname{sign}(\sigma)-L_{2} y .
$$

Define a new vector as $\vec{\gamma}=\left[\gamma_{1}, \gamma_{2}\right]^{\mathrm{T}}=\left[|\sigma|^{1 / 2} \operatorname{sign}(\sigma)\right.$, $\left.-L_{2} y\right]^{\mathrm{T}}$, we have $\vec{\gamma}^{2}=|\sigma|+L_{2}^{2} y^{2}$, sign $(\sigma)=\operatorname{sign}\left(\gamma_{1}\right)$, and $\left|\gamma_{1}\right|=|\sigma|^{1 / 2}$.

The time derivative of $\vec{\gamma}$ is

$$
\begin{aligned}
& \dot{\gamma}_{1}=\frac{1}{2}|\sigma|^{-1 / 2}\left(-L_{1} \gamma_{1}+\gamma_{2}+d\right), \\
& \dot{\gamma}_{2}=-L_{2} \dot{y}=-L_{2} \operatorname{sign}(\sigma)=-|\sigma|^{-1 / 2} L_{2} \gamma_{1} .
\end{aligned}
$$

So we have

$$
\begin{aligned}
& \dot{\vec{\gamma}}=\vec{A}_{\gamma} \vec{\gamma}+\vec{B}_{\gamma} d, \\
& \vec{A}_{\gamma}=|\sigma|^{-1 / 2}\left[\begin{array}{cc}
-\frac{L_{1}}{2} & \frac{1}{2} \\
-L_{2} & 0
\end{array}\right], \\
& \vec{B}_{\gamma}=|\sigma|^{-1 / 2}\left[\begin{array}{c}
\frac{1}{2} \\
0
\end{array}\right] .
\end{aligned}
$$

Select a Lyapunov candidate as

$$
V_{\mathrm{ob}}=\vec{\gamma}^{\mathrm{T}} \vec{P} \vec{\gamma}, \vec{P}=\left[\begin{array}{cc}
\lambda+\varepsilon^{2} & -\varepsilon \\
-\varepsilon & 1
\end{array}\right] \text {. }
$$

Since $\varepsilon$ and $\lambda$ are any positive constants, $\vec{P}$ is a positive definite matrix.

Its time derivative is

$$
\begin{aligned}
\dot{V}_{\mathrm{ob}} & =\dot{\vec{\gamma}}^{\mathrm{T}} \vec{P} \vec{\gamma}+\vec{\gamma}^{\mathrm{T}} \vec{P} \dot{\vec{\gamma}} \\
& =\vec{\gamma}^{\mathrm{T}}\left(\vec{P} \vec{A}_{\gamma}+\vec{A}_{\gamma}^{\mathrm{T}} \vec{P}\right) \vec{\gamma}+2 d \vec{B}_{\gamma}^{\mathrm{T}} \vec{P} \vec{\gamma} \\
& =-|\sigma|^{-1 / 2} \vec{\gamma}^{\mathrm{T}} \overrightarrow{\mathrm{Q}} \vec{\gamma}+2 d \vec{B}_{\gamma}^{\mathrm{T}} \vec{P} \gamma,
\end{aligned}
$$

where

$$
\vec{Q}=\left[\begin{array}{cc}
L_{1}\left(\lambda+\varepsilon^{2}\right)-2 L_{2} \varepsilon & L_{2}-\frac{L_{1}}{2} \varepsilon-\frac{1}{2}\left(\lambda+\varepsilon^{2}\right) \\
\star & \varepsilon
\end{array}\right] .
$$

Since

$$
2 \vec{B}_{\gamma}^{\mathrm{T}} \vec{P}=|\sigma|^{-1 / 2} \vec{G}, \vec{G}=\left[\lambda+\varepsilon^{2}-\varepsilon\right],
$$

(15) can be rewritten as

$$
\dot{V}_{\mathrm{ob}}=-|\sigma|^{-1 / 2}\left(\vec{\gamma}^{\mathrm{T}} \overrightarrow{\mathrm{Q}} \vec{\gamma}+d \vec{G} \vec{\gamma}\right) .
$$

We assume that the wave excitation force is bounded, so there is always a positive constant $\delta$ such that $|d| \leq \delta|\sigma|^{1 / 2}$ holds. It follows

$$
\dot{V}_{\mathrm{ob}}=-|\sigma|^{-1 / 2} \vec{\gamma}^{\mathrm{T}}(\vec{Q}+\vec{J}) \vec{\gamma}
$$

where

$$
\vec{J}=-|\sigma|^{-1 / 2}\left[\begin{array}{cc}
-\delta\left(\lambda+\varepsilon^{2}\right) & \frac{1}{2} \delta \varepsilon \\
\star & 0
\end{array}\right]
$$

Then, we have

$$
\vec{Q}+\vec{J}=\left[\begin{array}{cc}
\left(L_{1}-\delta\right)\left(\lambda+\varepsilon^{2}\right)-2 L_{2} \varepsilon & L_{2}-1 / 2 \varepsilon L_{1}-1 / 2\left(\lambda+\varepsilon^{2}\right)+1 / 2 \delta \varepsilon \\
\star & \varepsilon
\end{array}\right] .
$$


Substituting (10) into (21), it follows

$$
\vec{Q}+\vec{J}=\left[\begin{array}{cc}
L_{1} \lambda-(\varepsilon+\delta)\left(\lambda+\varepsilon^{2}\right) & \frac{1}{2} \delta \varepsilon \\
\star & \varepsilon
\end{array}\right] .
$$

Therefore, we have (23), if $L_{1} \geq\left((\varepsilon+\delta)\left(\lambda+\varepsilon^{2}\right)+\varepsilon / 2(\right.$ $\left.\left.\delta^{2}+1\right)\right) / \lambda$ holds.

$$
\begin{aligned}
\dot{V}_{\mathrm{ob}} & =-R V_{\mathrm{ob}}^{1 / 2}, \\
R & =\frac{\lambda_{\min }(\vec{Q}+\vec{J}) \lambda_{\min }^{1 / 2}(\vec{P})}{\lambda_{\max }(\vec{P})},
\end{aligned}
$$

where $\lambda_{\min }(\bullet)$ and $\lambda_{\max }(\bullet)$ denote the minimum and maximum eigenvalues of the matrix $\bullet$.

Since $\sigma \rightarrow 0$ holds if $\vec{\gamma} \rightarrow 0$, we have $h=x_{2}$ and $\dot{h=\dot{x}_{2}}$, which gives $d=\widehat{d}$ once $V_{\mathrm{ob}}=0$ holds.

It can be seen that the Lyapunov function $V_{\mathrm{ob}}$ is decreasing, which implies the attenuation of the estimation error by the proposed ADO.

By applying the finite-time stabilization theorem, the convergence time of the proposed $\mathrm{ADO}$ is

$$
T \leq \frac{2 V_{\mathrm{ob}}^{1 / 2}\left(t_{0}\right)}{R}
$$

\section{Multiple Surface Sliding Mode Controller}

In this section, a sliding mode-based control scheme for the motor driving system is designed considering both the tracking control and the synchronization control. Due to the strong robustness, the multiple sliding mode surface technique is applied to design the load tracking controller. The stability of the overall system is proven. Before the estimation error converges to zero, the tracking error converges within the neighbourhood of the origin. After the estimation error converges to zero, the tracking error converges to zero. The synchronization controller is developed based on the cross-coupled strategy.

4.1. Load Tracking Control. Since the model (8) is a secondorder nonlinear system with matched disturbances, two sliding surfaces are designed.

We firstly define the first sliding surface as

$$
\sigma_{1}=x_{1}-x_{1 d}
$$

where $x_{1 d}$ is the desired trajectory of $x_{1}$. The first reaching law is designed in a finite-time convergent form:

$$
\dot{\sigma}_{1}=-k_{1}\left|\sigma_{1}\right|^{\alpha} \operatorname{sign}\left(\sigma_{1}\right)
$$

where $k_{1}>0$ and $\alpha>1$ are constants.
To ensure that the relation between $\sigma_{1}$ and its time derivative $\dot{\sigma}_{1}$ holds, the second sliding surface is given as

$$
\sigma_{2}=\dot{\sigma}_{1}+k_{1}\left|\sigma_{1}\right|^{\alpha} \operatorname{sign}\left(\sigma_{1}\right)
$$

Its time derivative is

$$
\begin{aligned}
\dot{\sigma}_{2} & =\ddot{\sigma}_{1}+\alpha k_{1}\left|\sigma_{1}\right|^{\alpha-1} \dot{\sigma}=\dot{x}_{2}-\ddot{x}_{1 d}+\alpha k_{1}\left|\sigma_{1}\right|^{\alpha-1} \dot{\sigma} \\
& =J\left(u-n b_{m} x_{2}-f_{L}\left(x_{2}\right)\right)+d-\ddot{x}_{1 d}+\alpha k_{1}\left|\sigma_{1}\right|^{\alpha-1} \dot{\sigma}_{1}
\end{aligned}
$$

Theorem 2. For the system (8), the tracking error converges to zero with the following control law by using the ADO (9).

$$
u_{t}=J\left[\ddot{x}_{1 d}-\alpha k_{1}\left|\sigma_{1}\right|^{\alpha-1} \dot{\sigma}_{1}-k_{2} \sigma_{2}-\hat{d}\right]+n b_{m} x_{2}+f_{L}\left(x_{2}\right) \text {, }
$$

where $k_{2}>0$ is a constant.

Proof 2. Substituting (29) into (28), we have

$$
\dot{\sigma}_{2}=-k_{2} \sigma_{2}+d-\widehat{d}
$$

Expanding the vector by adding the second sliding surface $\sigma_{2}$ as an element, we have the close-loop vector as $\vec{\theta}=\left[\begin{array}{ll}\sigma_{2} & (C \vec{\gamma})^{T}\end{array}\right]^{\mathrm{T}}$, where $C$ is a positive constant.

Let $P_{e}=C^{2}$ and select a Lyapunov function of the overall system as

$$
V=\vec{\theta}^{\mathrm{T}} \vec{P}_{w} \vec{\theta}, \vec{P}_{w}=\frac{1}{2}\left[\begin{array}{cc}
P_{e} & 0 \\
0 & \vec{I}_{2}
\end{array}\right],
$$

where $P_{e}>0$ is the weighting parameter.

Its time derivative is

$$
\dot{V}=P_{e} \sigma_{2} \dot{\sigma}_{2}+\dot{V}_{\mathrm{ob}}=-P_{e} k_{2} \sigma_{2}^{2}+\dot{V}_{\mathrm{ob}}+\sigma_{2}(d-\widehat{d})
$$

From (23), we know that the estimation error cannot escape to infinity by the ADO, so we have a large enough constant $F$ such that $|d-\widehat{d}| \leq F$ holds, where $F>0$.

Then, (32) can be rewritten as

$$
\dot{V} \leq-P_{e} k_{2} \sigma_{2}^{2}-R V_{\mathrm{ob}}^{1 / 2}+\left|\sigma_{2}\right||d-\hat{d}| \leq-P_{e} k_{2} \sigma_{2}^{2}-R V_{\mathrm{ob}}^{1 / 2}+F\left|\sigma_{2}\right|
$$

Defining $\theta \in(0,1)$ as an arbitrary constant, we have

$$
\dot{V} \leq-(1-\theta) P_{e} k_{2} \sigma_{2}^{2}-R V_{\mathrm{ob}}^{1 / 2}+F\left|\sigma_{2}\right|-P_{e} k_{2} \theta \sigma_{2}^{2}
$$

So we have

$$
\dot{V} \leq-P_{e} k_{2}(1-\theta) \sigma_{2}^{2}, \text { if }\left|\sigma_{2}\right| \geq \frac{F}{P_{e} k_{2} \theta} .
$$


Therefore, the second sliding surface can converge within the neighbourhood of the origin: $D_{\sigma 2}=\left\{\sigma_{2}:\left|\sigma_{2}\right| \leq\right.$ $\left.F /\left(P_{e} k_{2} \theta\right)\right\}$.

Select a Lyapunov function as $V_{\sigma 1}=0.5 \sigma_{1}^{2}$, with (26), its time derivative is

$$
\dot{V}_{\sigma 1}=\sigma_{1} \dot{\sigma}_{1}=-k_{1}\left|\sigma_{1}\right|^{\alpha+1}=-k_{1} 2^{\alpha+1 / 2} V^{\alpha+1 / 2}
$$

From (27) and (35), we have

$$
\dot{\sigma}_{1}+k_{1} \sigma_{1}^{\alpha}=\phi
$$

where $\phi \leq \Phi=F /\left(P_{e} k_{2} \theta\right)$.

From (37), we have

$$
\dot{V}_{\sigma 1} \leq 0, \text { if } 1-\frac{\phi}{k_{1} \sigma_{1}^{\alpha}}>0
$$

So the position tracking error converges within the neighbourhood of the origin

$$
\left|\sigma_{1}\right| \leq\left(\frac{\Phi}{k_{1}}\right)^{1 / 2}
$$

and velocity tracking error converges within the neighbourhood of the origin

$$
\left|\dot{\sigma}_{1}\right| \leq k_{1}\left|\sigma_{1}\right|^{2}+|\phi| \leq 2 \Phi
$$

So before $\mathrm{ADO}$ completes its convergence, the overall system is stable, and the tracking error is decreasing since the estimation error is decreasing. After the ADO completes its convergence, i.e., the estimation error is zero if $t>T$, we have $F=0$, which implies $\Phi=0$. So the tracking error converges to zero.

Therefore, the whole system is stable by using the proposed ADO (9) and the proposed controller (29).

4.2. Dual-Motor Synchronization Control. To the dual-motor driving system, the synchronization of driving motors is essential. Even if the driving motors are chosen as the same type, the position error exists due to the gear clearance, measurement errors, and external disturbances. To avoid the potential motor collision, a synchronization control strategy is developed embedded within the tracking controller of the dual-motor driving system.

Design a sliding mode surface as

$$
\sigma_{s 1}=\theta_{1}-\theta_{2}
$$

The reaching law is designed as

$$
\dot{\sigma}_{s 1}=-k_{s 1}\left|\sigma_{s 1}\right|^{\alpha_{s}} \operatorname{sgn}\left(\sigma_{s 1}\right)
$$

where $\alpha_{s} \geq 1, k_{s 1}>0$.
With the similar procedure of the tracking controller design, the second sliding surface is expressed as

$$
\dot{\sigma}_{s 2}=\ddot{\sigma}_{s 1}+\alpha_{s} k_{s 1}\left|\sigma_{s 1}\right|^{\left(\alpha_{s}-1\right)} \dot{\sigma}_{s 1}
$$

According to dual-motor system (1), we have

$$
\begin{aligned}
\dot{\sigma}_{s 2}= & \frac{1}{J_{m}}\left(u_{1}-\tau_{1}-b_{m} \dot{\theta}_{1}\right)-\frac{1}{J_{m}}\left(u_{2}-\tau_{2}-b_{m} \dot{\theta}_{2}\right) \\
& +\alpha_{s} k_{s 1}\left|\sigma_{s 1}\right|^{\left(\alpha_{s}-1\right)} \dot{\sigma}_{s 1} .
\end{aligned}
$$

The synchronization controller is designed as follows:

$$
u_{\mathrm{si}}=\frac{(-1)^{i+1} J_{m}}{2}\left(-\alpha_{s} k_{s 1}\left|\sigma_{s 1}\right|^{\left(\alpha_{s}-1\right)} \dot{\sigma}_{s 1}-k_{s 2} \sigma_{s 2}\right)+\tau_{i}+b_{m} \dot{\theta}_{i},
$$

where $k_{s 2}>0$ is the tuning parameter.

The overall controller considering both tracking and synchronization problem is developed as follows:

$$
u_{i}=\Xi u_{\mathrm{si}}+u_{t}
$$

where $\Xi$ is the synchronization coefficient and expressed as

$$
\Xi=1-\frac{1}{\exp \psi\left|\sigma_{s 1}\right|},
$$

where $\psi$ is a positive parameter.

Remark 1. The synchronization extent between two driving motors can be described by the designed synchronization coefficient $\Xi$. When the two driving motors are not synchronized because of the gear clearance and the external disturbance, $\Xi$ converges to 1 which makes the synchronization controller accounts. Then, the position error convergence can be guaranteed by the designed synchronization controller (45). On the other hand, when the position error $\sigma_{s 1} \rightarrow$ $0, \Xi \rightarrow 0$ and the load tracking control will not be influenced. Therefore, the load tracking problem can be dealt with the proposed controller $u_{i}$ while considering the synchronization problem of driving motors.

\section{Simulation Results}

The system parameters and controller parameters are shown in Tables 1 and 2. Contrastive results are demonstrated to illustrate the efficacy of the proposed method.

5.1. Disturbance Observer Verification. To show the effectiveness of the proposed disturbance observer, $d=0.2 \sin (t) \mathrm{rad}$ is chosen as an equivalent external disturbance in (8). The observation performance and the observation error are shown in Figures 3 and 4.

We can see from Figure 3 that with the designed observer, the external disturbance can be achieved precisely. The high convergence speed illustrates the advantage of the finitetime observer. In Figure 4, the observation error is relatively 
TABle 1: Parameters of dual-motor driving system.

\begin{tabular}{lcc}
\hline Parameter & Value & Unit \\
\hline$J_{m}$ & 0.0026 & $\mathrm{~kg} \cdot \mathrm{m}^{2}$ \\
$J_{L}$ & 0.0113 & $\mathrm{~kg} \cdot \mathrm{m}^{2}$ \\
$n$ & $70 / 6$ & $/$ \\
$b_{m}$ & 0.015 & $\mathrm{Nm} \cdot \mathrm{s} / \mathrm{rad}$ \\
$k$ & 560 & $\mathrm{Nm} / \mathrm{rad}$ \\
$c$ & 0.15 & $\mathrm{Nm} \cdot \mathrm{s} / \mathrm{rad}$ \\
$d_{c}$ & 0.2 & $\mathrm{Rad}$ \\
\hline
\end{tabular}

TABle 2: Controller and observer parameters.

\begin{tabular}{lcccc}
\hline Parameter & $k_{1}$ & $k_{2}$ & $k_{s 1}$ & $k_{s 2}$ \\
\hline Value & 20 & 15.5 & 10 & 9 \\
Parameter & $\alpha$ & $\alpha_{s}$ & $\psi$ & $\varepsilon$ \\
Value & 4 & 2.5 & 1.5 & 15 \\
Parameter & $\lambda$ & $k_{p}$ & $k_{i}$ & $k_{d}$ \\
Value & 12 & 20 & 8 & 4 \\
\hline
\end{tabular}

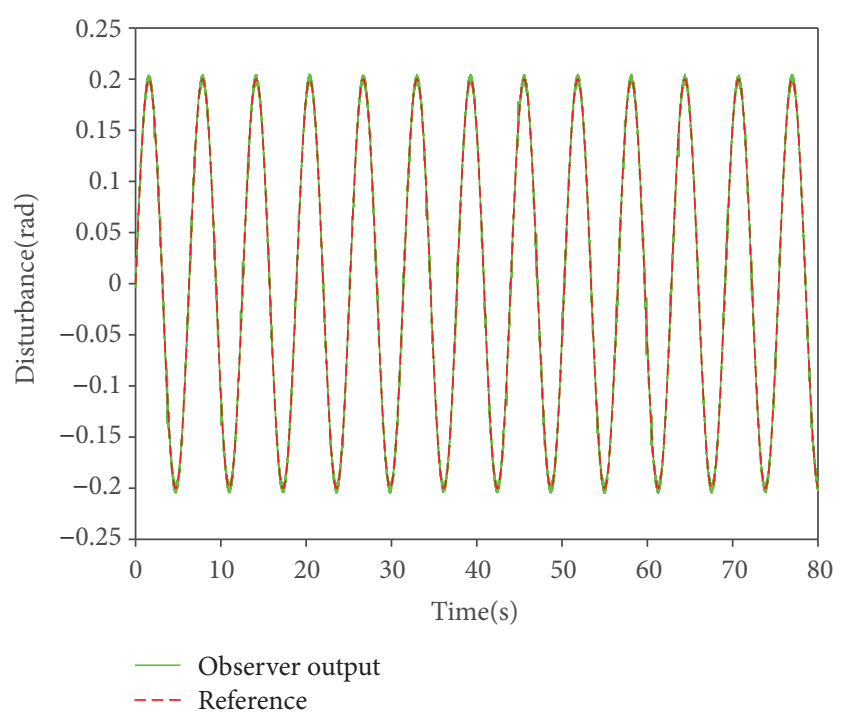

FIgURE 3: Observation performance.

small. The maximum estimation error is $15 \%$ and the most estimation errors are located within $0.01 \mathrm{rad}$.

5.2. Controller Verification. A sinusoidal signal $y_{d}=2 \sin (t)$ rad is chosen as a reference signal in model (1). With parameters shown in Table 2, contrastive results with and without disturbance compensation are shown in Figures 5-7.

From Figures 5 to 7, we can see that with the proposed control scheme, the load can be driven to track the reference signal. With the disturbance compensation based on the developed disturbance observer, the better control performance and the smaller tracking error can be achieved. In Figure 6, with the disturbance compensation, the maximum tracking error is $4 \%$. Hence, the precise tracking control can be achieved. To further demonstrate the advantage of the proposed controller, the PID control which has been

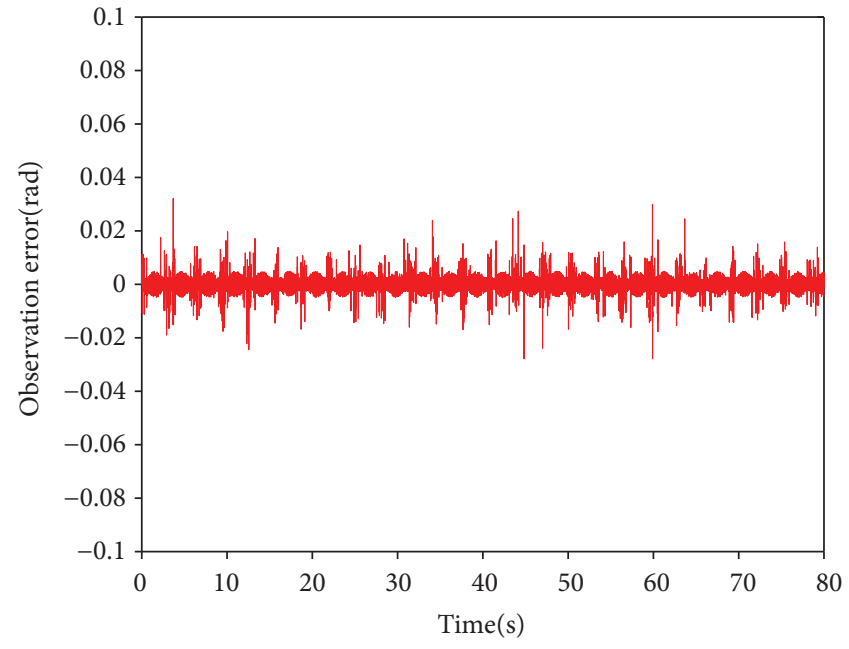

Figure 4: Observation error.

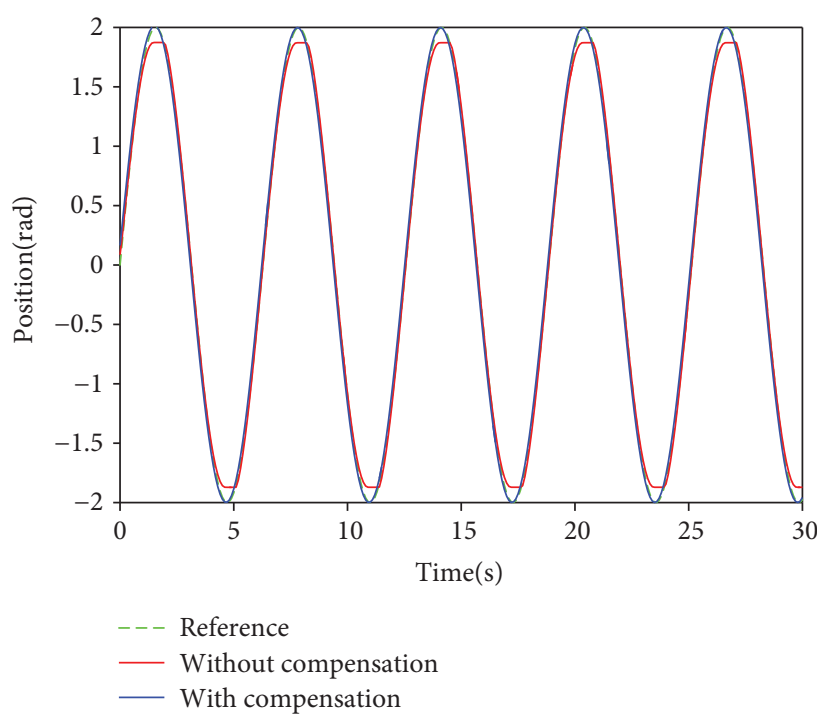

FIGURE 5: Tracking performance.

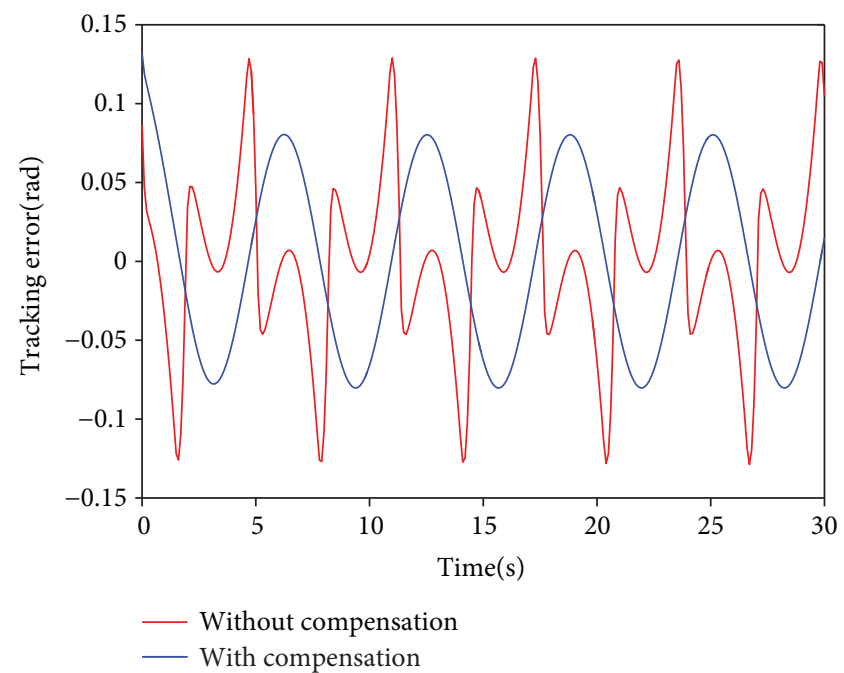

Figure 6: Tracking error. 


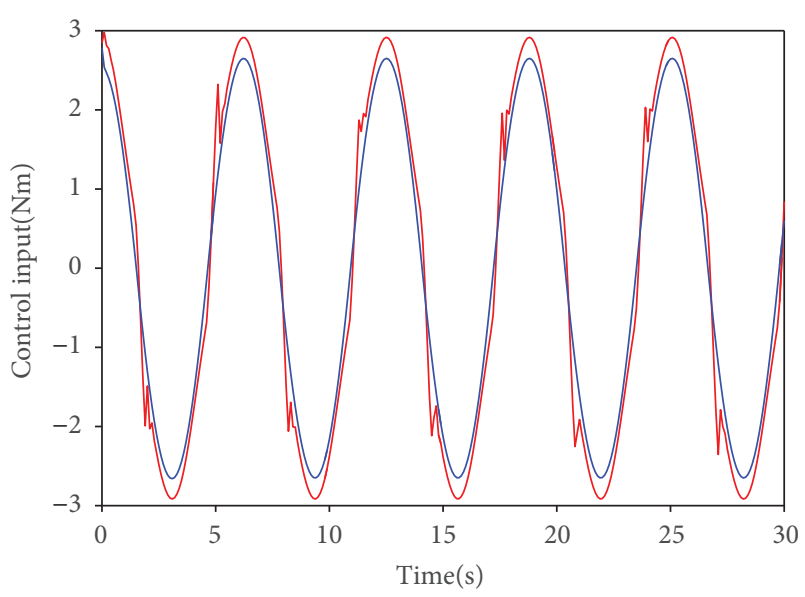

Without compensation

- With compensation

Figure 7: Control input.
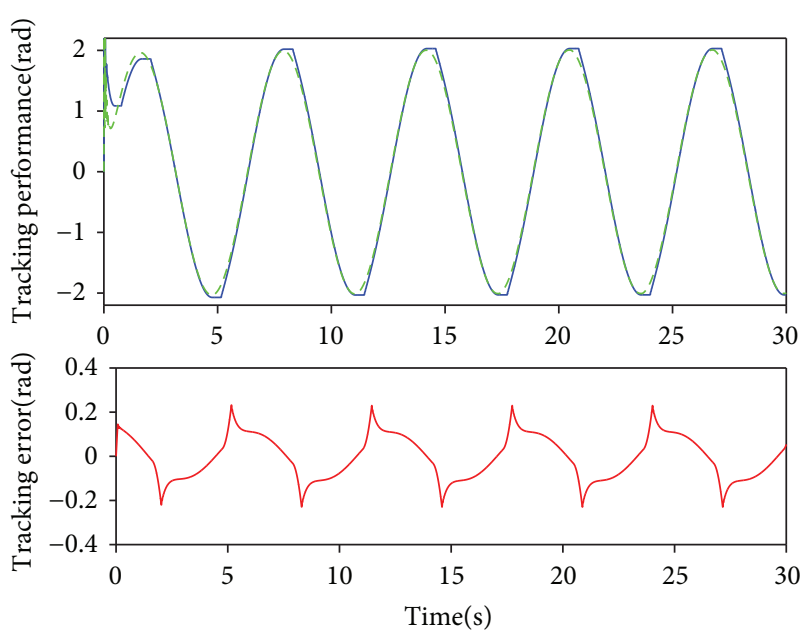

_ PID tracking performance

- - Reference

FIGURE 8: Tracking performance of PID.

widely used in the control of motor driving system is introduced for comparison. The control performance of the PID controller with parameters shown in Table 2 is shown in Figure 8.

From Figure 8, we can see that the largest tracking error is $0.2 \mathrm{rad}$. Hence, better dynamic and steady state performance with smaller tracking error can be achieved when using the proposed controller. Due to the structure of dual-motor driving system, the satisfactory control performance can be achieved based on the synchronization of the two driving motors. The trajectories of the two driving motors are shown in Figure 9.

In Figure 9, we can see that with the developed synchronization controller, a satisfactory synchronization performance is achieved without any influences on the tracking performance. Therefore, the effectiveness of the proposed disturbance-based control scheme for dual-motor driving system is demonstrated.

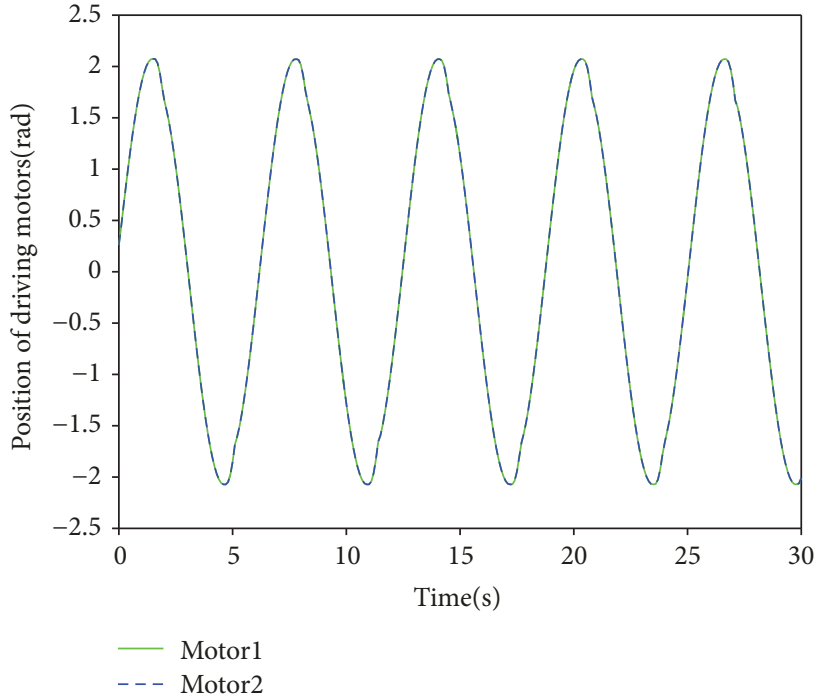

FIgURe 9: Positions of driving motors.

\section{Conclusions}

In this paper, the high accuracy tracking control problem of the dual-motor driving system was investigated. A sliding mode control scheme based on adaptive disturbance observer was developed. To deal with the unknown matched disturbance, a finite-time convergent adaptive observer was proposed. The sliding mode controller based on the multisurface technique was designed considering both the tracking and the synchronization problems. The stability of the overall system including both the observer and the controller was analyzed. The tracking error was within the neighbourhood of the origin before the observer completes its convergence and converges to zero thereafter. Simulation results demonstrated the efficacy of the proposed finite-time observer and the sliding mode controller. The finite-time controller design method for the high-order system can be considered to avoid the model uncertainty in the future work.

\section{Data Availability}

The data used to support the findings of this study are available from the corresponding author upon request.

\section{Conflicts of Interest}

The authors declare that they have no conflicts of interest.

\section{Acknowledgments}

This work is sponsored by National Natural Science Foundation of China (no. 61433003, no. 61273150 and no. 61321002).

\section{References}

[1] S. Wang, X. Ren, J. Na, and T. Zeng, "Extended-state-observerbased funnel control for nonlinear servomechanisms with 
prescribed tracking performance," IEEE Transactions on Automation Science and Engineering, vol. 14, no. 1, pp. 98-108, 2017.

[2] S. Wang, J. Na, and X. Ren, "Rise-based asymptotic prescribed performance tracking control of nonlinear servo mechanisms," IEEE Transactions on Systems, Man, and Cybernetics: Systems, no. 99, pp. 1-12, 2017.

[3] J. Na, M. N. Mahyuddin, G. Herrmann, X. Ren, and P. Barber, "Robust adaptive finite-time parameter estimation and control for robotic systems," International Journal of Robust and Nonlinear Control, vol. 25, no. 16, pp. 3045-3071, 2015.

[4] S.-M. Lu and D.-J. Li, "Adaptive neural network control for nonlinear hydraulic servo-system with time-varying state constraints," Complexity, vol. 2017, Article ID 6893521, 11 pages, 2017.

[5] Q. Chen, X. Ren, J. Na, and D. Zheng, “Adaptive robust finitetime neural control of uncertain PMSM servo system with nonlinear dead zone," Neural Computing and Applications, vol. 28, no. 12, pp. 3725-3736, 2017.

[6] R. Ghazali, Y. M. Sam, M. F. Rahmat, A. W. I. M. Hashim, and Zulfatman, "Sliding mode control with PID sliding surface of an electro-hydraulic servo system for position tracking control," Australian Journal of Basic and Applied Sciences, vol. 4, no. 10, pp. 4749-4759, 2010.

[7] D. Sun, X. Shao, and G. Feng, "A model-free cross-coupled control for position synchronization of multi-axis motions: theory and experiments," IFAC Proceedings Volumes, vol. 38, no. 1, pp. 1-6, 2005.

[8] D. Sun, "Position synchronization of multiple motion axes with adaptive coupling control," Automatica, vol. 39, no. 6, pp. 997-1005, 2003.

[9] Z. Deng, J. Shang, and X. Nian, "Synchronization controller design of two coupling permanent magnet synchronous motors system with nonlinear constraints," ISA Transactions, vol. 59, pp. 243-255, 2015.

[10] B. Jiang, Q. Hu, and M. I. Friswell, "Fixed-time attitude control for rigid spacecraft with actuator saturation and faults," IEEE Transactions on Control Systems Technology, vol. 24, no. 5, pp. 1892-1898, 2016.

[11] C. Edwards and S. Spurgeon, Sliding Mode Control: Theory and Applications, CRC Press, 1998.

[12] V. I. Utkin, J. Guldner, and J. Shi, Sliding Mode Control in Electro-Mechanical Systems. 1em plus $0.5 \mathrm{em}$ minus $0.4 \mathrm{em}$, CRC Press, 2009.

[13] Y. Shtessel, C. Edwards, L. Fridman, and A. Levant, Sliding Mode Control and Observation. 1em plus 0.5em minus 0.4em, Springer New York, 2014.

[14] A. T. Azar and Q. Zhu, Advances and Applications in Sliding Mode Control Systems. 1em plus 0.5em minus 0.4em, Springer New York, 2016.

[15] R. Furfaro, D. Cersosimo, and D. R. Wibben, "Asteroid precision landing via multiple sliding surfaces guidance techniques," Journal of Guidance, Control, and Dynamics, vol. 36, no. 4, pp. 1075-1092, 2013.

[16] Z. Song, H. Li, and K. Sun, "Finite-time control for nonlinear spacecraft attitude based on terminal sliding mode technique," ISA Transactions, vol. 53, no. 1, pp. 117-124, 2014.

[17] B.-Z. Guo and H.-C. Zhou, "The active disturbance rejection control to stabilization for multi-dimensional wave equation with boundary control matched disturbance," IEEE Transactions on Automatic Control, vol. 60, no. 1, pp. 143-157, 2015.
[18] W.-H. Chen, D. J. Ballance, P. J. Gawthrop, and J. O’Reilly, "A nonlinear disturbance observer for robotic manipulators," IEEE Transactions on Industrial Electronics, vol. 47, no. 4, pp. 932-938, 2000.

[19] E. Sariyildiz and K. Ohnishi, "Stability and robustness of disturbance-observer-based motion control systems," IEEE Transactions on Industrial Electronics, vol. 62, no. 1, pp. 414422, 2015.

[20] M. Chen, P. Shi, and C.-C. Lim, "Robust constrained control for MIMO nonlinear systems based on disturbance observer," IEEE Transactions on Automatic Control, vol. 60, no. 12, pp. 3281-3286, 2015.

[21] B. Xu, F. Sun, Y. Pan, and B. Chen, "Disturbance observer based composite learning fuzzy control of nonlinear systems with unknown dead zone," IEEE Transactions on Systems, Man, and Cybernetics: Systems, vol. 47, no. 8, pp. 1854-1862, 2017.

[22] S. Mobayen and S. Javadi, "Disturbance observer and finitetime tracker design of disturbed third-order nonholonomic systems using terminal sliding mode," Journal of Vibration and Control, vol. 23, no. 2, pp. 181-189, 2017.

[23] J. Na, X. Ren, and D. Zheng, "Adaptive control for nonlinear pure-feedback systems with high-order sliding mode observer," IEEE Transactions on Neural Networks and Learning Systems, vol. 24, no. 3, pp. 370-382, 2013.

[24] X. Jiao, J. Zhang, and T. Shen, "An adaptive servo control strategy for automotive electronic throttle and experimental validation," IEEE Transactions on Industrial Electronics, vol. 61, no. 11, pp. 6275-6284, 2014. 


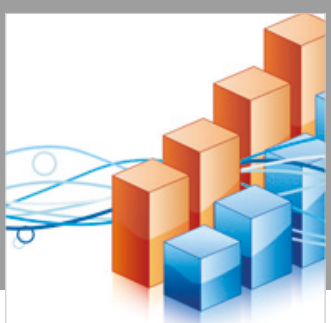

Advances in

Operations Research

\section{-n-m}
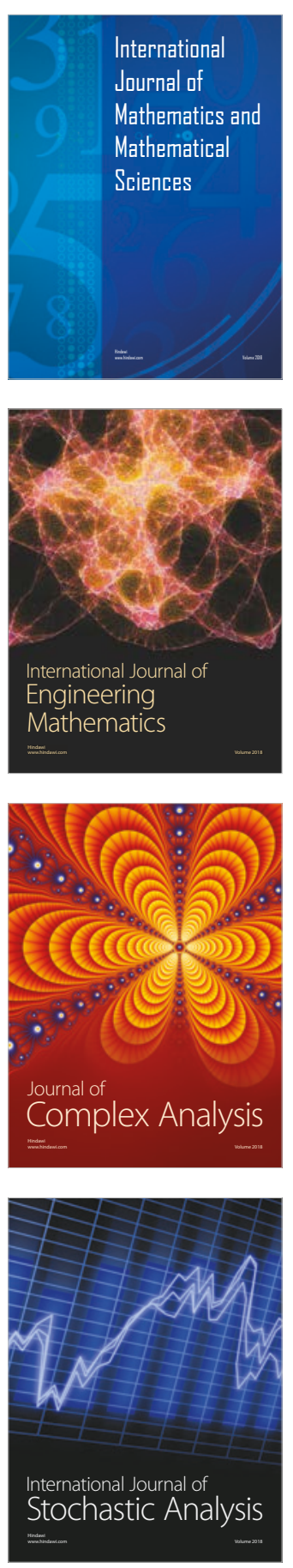
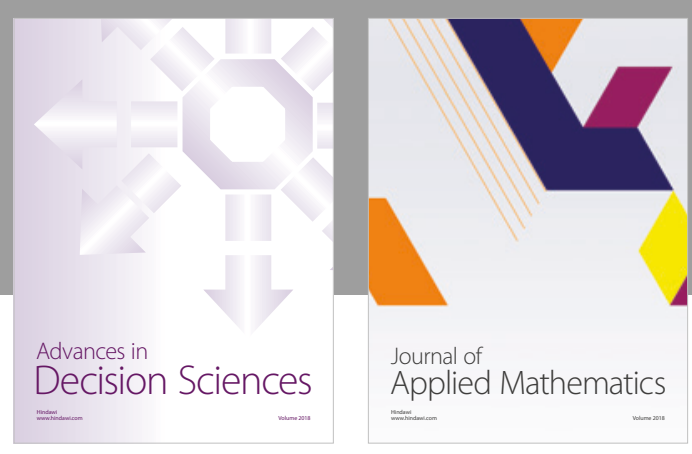

Journal of

Applied Mathematics
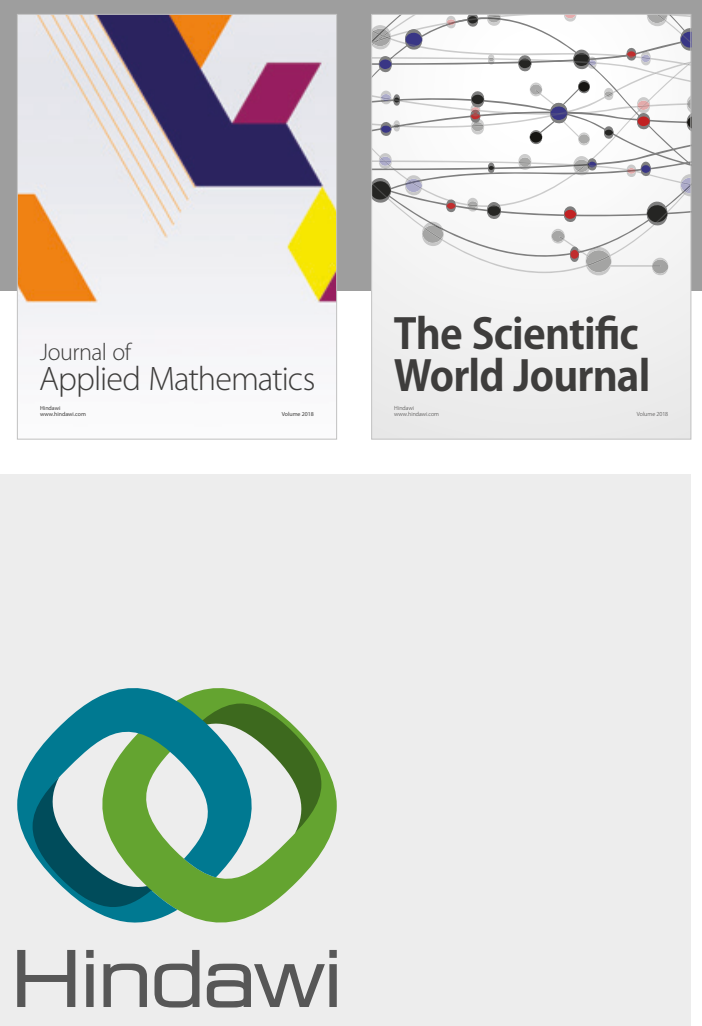

Submit your manuscripts at

www.hindawi.com

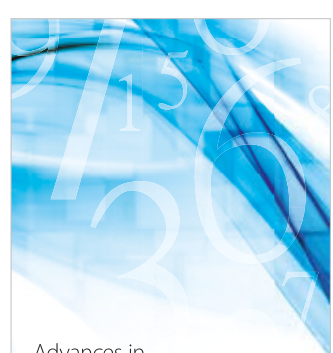

Advances in
Numerical Analysis
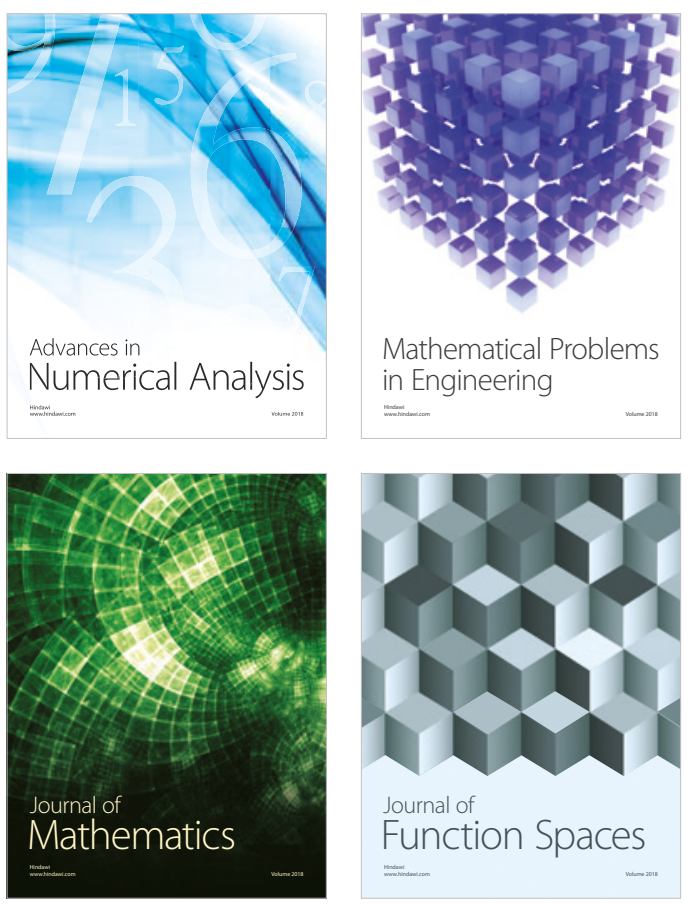

Mathematical Problems in Engineering

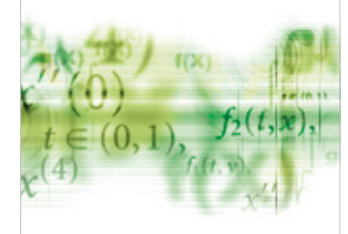

International Journal of

Differential Equations

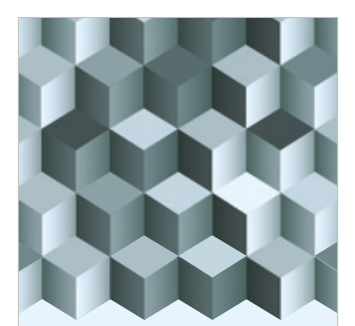

Journal of

Function Spaces
The Scientific

World Journal

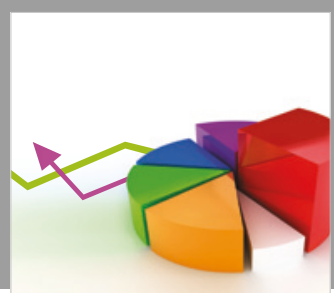

Journal of

Probability and Statistics
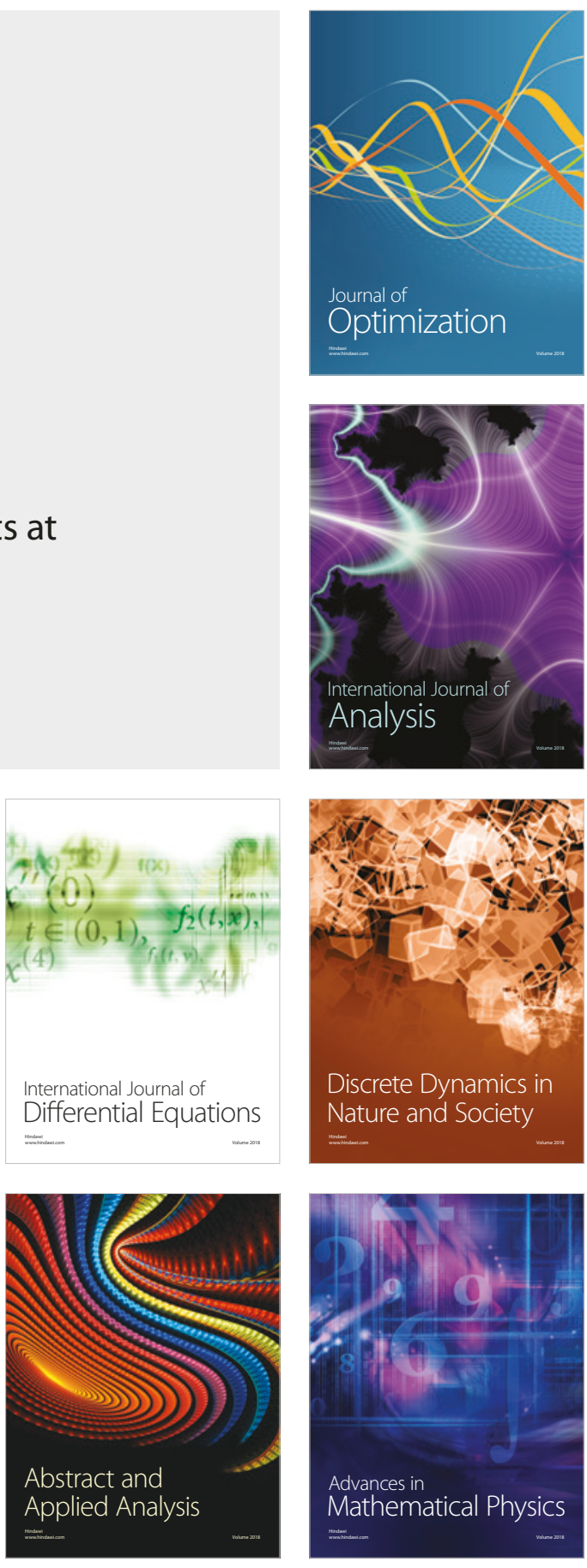